\title{
FBG Optimization Using Spline Encoded Evolution Strategy
}

\author{
M. J. de Sousa, J. C. W. A. Costa, R. M. de Souza, R. V. M. P. Pantoja \\ Federal University of Pará(UFPA), marcojsousa@ufpa.br, jweyl@ufpa.br, rmedeiros@ufpa.br, \\ rvillar@ufpa.br
}

\begin{abstract}
This paper presents an encoding scheme adapted for Fiber Bragg Grating (FBG) optimization using metaheuristics. The proposed encoding scheme uses spline approximations in order to build softened refractive index profiles from few encoded parameters. This approach is suitable for Fiber Bragg Grating (FBG) synthesis because it ensures both the reduction of the problem dimensionality and the respect of important restrictions associated to the FBG manufacture. Simulations are shown where an ES using the spline encoding was able to converge faster and produce more interesting filters, when compared with conventional encoding schemes.
\end{abstract}

Index Terms - FBG, Evolution Strategies, spline, chromosome, encoding.

\section{INTRODUCTION}

Fiber Bragg Gratings (FBGs) are flexible components. By adjusting the parameters that describes their refractive index profile, it is possible to obtain filters showing reflectance spectrum adapted to almost any type of application. Unfortunately, this procedure (FBG synthesis) is not a trivial problem and several techniques have been proposed with some degree of success.

For example, if only weak gratings are considered, the refractive index profile can be obtained from the Fourier Transform of the reflection coefficient. This was the approach used by Winick and Roman [1] with interesting but inaccurate results. On the other hand, other techniques like these based on Layer Peeling (LP) [2] [3] are capable of very accurate solutions in terms of reflectance spectrum, but have as side effect the generation of large and complex refractive index profiles that are difficult to built using Ultra Violet (UV) mask manufacture techniques [4]. In order to attend all restrictions associated to the FBG manufacture, Askanes et al [5] proposed a hybrid method that combines classical optimization techniques and the LP algorithm.

Other interesting design alternatives are those based on Evolutionary Algorithms (EA). Genetic Algorithm (GA) [6] and Particle Swarm Optimization (PSO) [7] allow achieving satisfactory results even under heavy restrictions imposed by the manufacture process. For example, in [8], a pioneer article about the use of GAs for FBG synthesis, feasible Wavelength Division Multiplexing (WDM) filters with negligible dispersion are obtained. In [9], the FBG synthesis is performed using the Covariance Matrix Adapted Evolution Strategy (CMAES) with relative great success. Similar results are achieved in [10] using PSO. In [11], a GA is applied in the synthesis of Triangular FBGs (TFBGs). In [12], the TFBG synthesis is performed again, but using the CMAES. 
Although literature shows that metaheuristics can be used to synthesize FBGs, they suffer because of the high number of parameters needed to represent a FBG properly. Using the modeling based on uniform sections presented in [13], an apodized FBG one centimeter long could be well represented by about one hundred uniform sections, each one modeled by four parameters. Even considering a single parameter by section, about one hundred in total must be used to represent the entire FBG. This high dimensionality can make the metaheuristic optimization scheme not practical. Frequently in the literature a FBG is designed using a reduced number of uniform sections. For example, in [10], only 20 sections are considered. The use of a reduced number of sections can generate mismatches large enough to work like mirrors inside the FBG, which can create several cavities along the grating. In this case the reflectance spectrum can result noisy and full of undesired lobes, affecting the metaheuristic convergence.

Therefore, it is interesting to produce softened refractive index profiles and find a way to do that using as few parameters as possible. Fortunately, according to the information theory, softened signal formats shall carry naturally less information and, consequently, it is possible to use less parameter to encode them. This article explores the use of spline approximations to create softened profiles from few points stored in ES individuals (chromosomes) [14]-[16]. Section II presents the basic FBG model. Section III presents the usual way to encode a FBG and the proposed spline encodings. Section IV presents the ES algorithm. Section V compares the performance of several encoding schemes through computer simulations. Finally, Section VI presents the conclusions.

\section{FBG MODEL}

Following the matrix formulation from [13], the refractive index profile is given in function of the axial distance $z$ :

$$
n(z)=n_{e f f}+\delta n \cos \left(\frac{4 \pi n_{e f f} z}{\lambda_{B}}\right),
$$

where $n_{\text {eff }}$ is the effective refractive index, $\delta n$ the modulation parameter and $\lambda_{B}$ is Bragg wavelength.

Using the coupled mode theory, it is possible to calculate a $2 \times 2$ matrix $\mathbf{F}$ that represents a uniform section of length $\Delta z$ :

$$
\left[\begin{array}{l}
R(\Delta z) \\
S(\Delta z)
\end{array}\right]=\mathbf{F} \times\left[\begin{array}{c}
R(0) \\
S(0)
\end{array}\right],
$$

where $R(z)$ and $S(z)$ stand for the field amplitude functions of the propagating and back propagating modes respectively. Hence, $R(0)$ and $S(0)$ represent the field amplitudes before the section at $\mathrm{z}=0$ and, at $z=\Delta z, R(\Delta z)$ and $S(\Delta z)$ represent the field amplitudes after the section. The transfer matrix $\mathbf{F}$ is calculated in function of $\delta n, \lambda_{B}, \Delta z$, and $n_{\text {eff }}$, all of them considered constant along the section. Indeed, in this work, $n_{\text {eff }}$ will be considered always constant for the entire grating. 
A non uniform Bragg Grating can be modeled as a sequence of $M$ uniform short gratings (uniform sections). Let $\mathbf{F}_{k}$ be the transfer matrix of the $k$-th section, where $k=0$ for the first section at $z=0$. The total transfer matrix for the apodized grating of length $L=M \times \Delta z$ can be obtained by multiplying all its $M$ section matrices:

$$
\mathbf{F}_{T}=\mathbf{F}_{M-1} \times \mathbf{F}_{M-2} \ldots \times \mathbf{F}_{k} \times \mathbf{F}_{k-1} \ldots \times \mathbf{F}_{1} \times \mathbf{F}_{0} .
$$

Now (2) can be rewritten in function of the total matrix $\mathbf{F}_{T}$ :

$$
\left[\begin{array}{c}
R(L) \\
S(L)
\end{array}\right]=\mathbf{F}_{T} \times\left[\begin{array}{c}
R(0) \\
S(0)
\end{array}\right]=\left[\begin{array}{ll}
F_{T_{11}} & F_{T_{12}} \\
F_{T_{21}} & F_{T_{22}}
\end{array}\right] \times\left[\begin{array}{c}
R(0) \\
S(0)
\end{array}\right],
$$

where $R(L)$ and $S(L)$ represent respectively the field amplitudes of the propagating and back propagating modes at $z=L$.

The FBG reflectance is given in function of $\mathbf{F}_{T}$ elements by:

$$
R=\left(\frac{\left|F_{T_{21}}\right|}{\left|F_{T_{22}}\right|}\right)^{2}
$$

An apodized FBG can be represented in a vector form like $\left(\delta n_{0}, \delta n_{1}, \ldots, \delta n_{k}, \ldots, \delta n_{M-2}, \delta n_{M-1}, \lambda_{\mathrm{B} 0}, \lambda_{\mathrm{B} 1}\right.$, $\left.\ldots, \lambda_{\mathrm{B} k}, \ldots, \lambda_{\mathrm{B} M-2}, \lambda_{\mathrm{B} M-1}, \Delta z_{0}, \Delta z_{1}, \ldots, \Delta z_{k}, \Delta z_{M-2}, \Delta z_{M-1}\right)$, which is also a natural way to represent a FBG as an individual in metaheuristics. In practical terms, not all parameters should be present in the vector because they could be constant or known for the entire grating. For example, the representation in the format ( $\left.\delta n_{0}, \delta n_{1}, \ldots, \delta n_{k-1}, \delta n_{k}, \delta n_{k+1}, \ldots, \delta n_{M-2}, \delta n_{M-1}\right)$ was essentially the way chosen in [12] to represent individuals in the CMAES.

\section{ENCODING STRATEGIES}

Let us consider to be enough to represent ES individuals as $\mathbf{Y}=\left(y_{0}, y_{1}, \ldots, y_{k-1}, y_{k}, y_{k+1}, \ldots, y_{M-2}\right.$, $y_{M-1}$ ), where $y_{k}$ could be any parameter among $\delta n_{k}, \lambda_{\mathrm{B} k}$ or $\Delta z_{k}$. This encoding scheme will be referred here as direct encoding (DE), where the number of search space dimensions is equal to $M$, the same number of uniform sections. Satisfactory FBG representations make use of at least 20 sections for a centimeter long grating [10]-[12].

An interesting way to reduce the number of dimensions is through some sort of indirect encoding, where the individuals store few parameters in a vector form like $\boldsymbol{X}=\left(x_{0}, x_{1}, \ldots, x_{j-1}, x_{j}, x_{j+1}, \ldots, x_{m-2}\right.$, $\left.x_{m-1}\right)$ with $m<M$. Any $y_{k}$ can be calculated from $\boldsymbol{X}$ using linear interpolation:

$$
y_{k}=x_{j}+\left(x_{j}-x_{j}\right)\left(d_{k}-j\right)
$$

where

$$
d_{k}=\frac{m \times k}{M-1}
$$

And $j=$ floor $\left(d_{k}\right)$, with the function floor( ) returning the closest integer below the argument. The 
encoding scheme given by (6) and (7) will be referred as linear encoding or LE.

Another approach is to replace the linear interpolation by a spline approximation. This paper explores quadratic and cubic splines [16]. For the quadratic spline encoding (QSE), $y_{k}$ can be calculated from $\boldsymbol{X}$ by

$$
y_{k}= \begin{cases}x_{j}+\left(x_{j+1}-x_{j}\right)\left[d_{k}-\text { floor }\left(d_{k}\right)\right], & j=0 \\ x_{j-1}+\left(x_{j}-x_{j-1}\right)\left[d_{k}-\text { floor }\left(d_{k}\right)\right], & j=m-1 \\ 0.5\left[(1-t)^{2} x_{j-1}+t^{2} x_{j+1}\right]+[t(1-t)+0.5] x_{j}, & \text { otherwise }\end{cases}
$$

where $d_{k}$ is given by (7), $j=\operatorname{round}\left(d_{k}\right)$ and $t=d_{k}-j+1 / 2$. The function round( ) returns the nearest integer from the argument.

For the cubic spline encoding (CSE), the relationship between $y_{k}$ and $\boldsymbol{X}$ is given by:

$$
y_{k}=A(1-t)^{3}+3 B t(1-t)^{2}+3 C t^{2}(1-t)+D t^{3}
$$

where $d_{k}$ is given by (7), $j=\operatorname{trunc}\left(d_{k}\right)$ and $t=d_{k}-j$. The parameters $A, B, C$ and $D$ are given respectively by:

$$
\begin{gathered}
A=\left\{\begin{array}{ll}
\frac{1}{6}\left[x_{j-1}+4 x_{j}+x_{j+1}\right], & 0<j<(m-1) \\
x_{j}, & \text { otherwise }
\end{array},\right. \\
B= \begin{cases}\frac{1}{3}\left(2 x_{j}+x_{j+1}\right), & j<(m-1) \\
x_{j}, & \text { otherwise }\end{cases} \\
C= \begin{cases}\frac{1}{3}\left(x_{j}+2 x_{j+1}\right), & j<(m-1) \\
x_{m-1}, & \text { otherwise }\end{cases}
\end{gathered}
$$

and

$$
D=\left\{\begin{array}{ll}
\frac{1}{6}\left(x_{j}+4 x_{j+1}+x_{j+2}\right), & j<m-3 \\
x_{m-1}, & \text { otherwise }
\end{array} .\right.
$$

For $m<3$ the cubic spline approximation shall be replaced by linear interpolation and $y_{k}$ shall be calculated using (6) and (7).

Examples of curves constructed by using LE, QSE and CSE are shown in the Fig. 1. The vector $(0.0,0.25,0.1,0.7,1.0,0.7,0.1,0.25,0.0)$ with $m=9$ was used to produce these curves, each one with 200 points $(M=200)$. As one can see, the spline approximations are smoother than LE and the CSE is the most.

\section{EVOLUTION STRATEGY}

Evolution Strategies, among other meta-heuristics like GA, evolve individuals applying nature inspired operators. Back in 1970s, ES used to evolve a single individual by creating only one child 
each generation [17]. Modern versions of ES use a population of parents and differ from GA basically in the encoding and selection schemes [18].

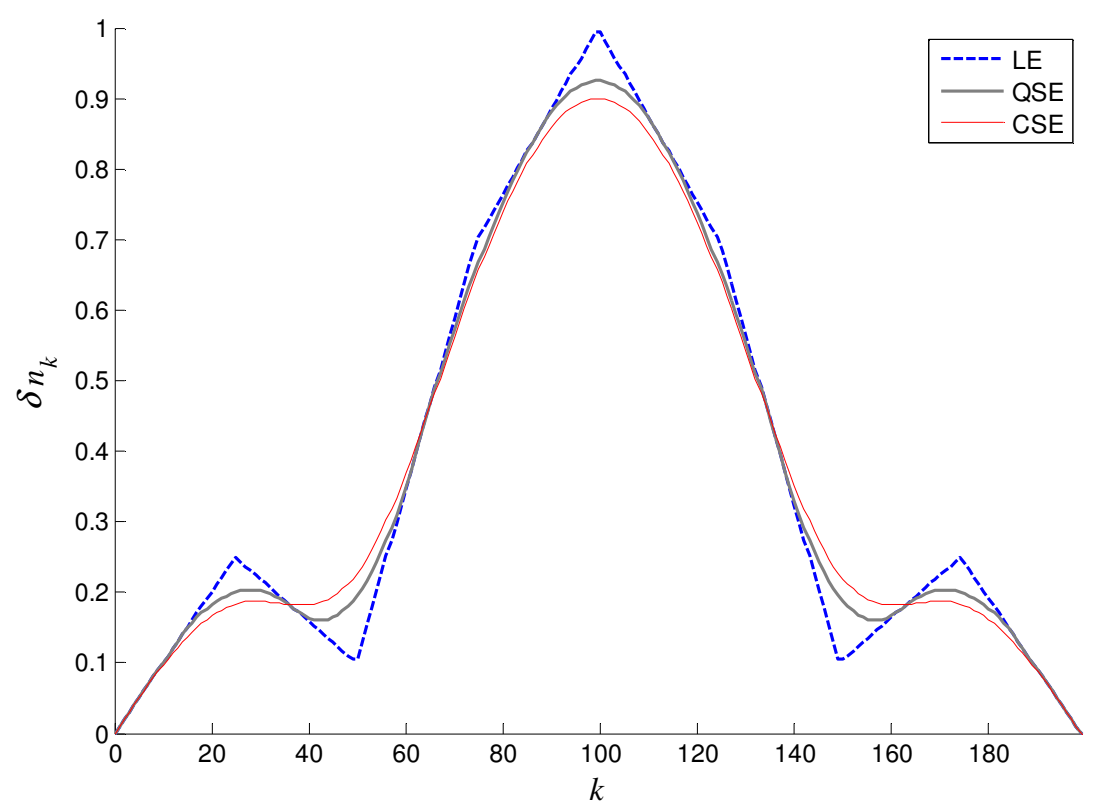

Fig. 1. $\delta n_{k}$ profiles calculated using LE, QSE and CSE for $M=200$, obtained from a test curve with $m=9$.

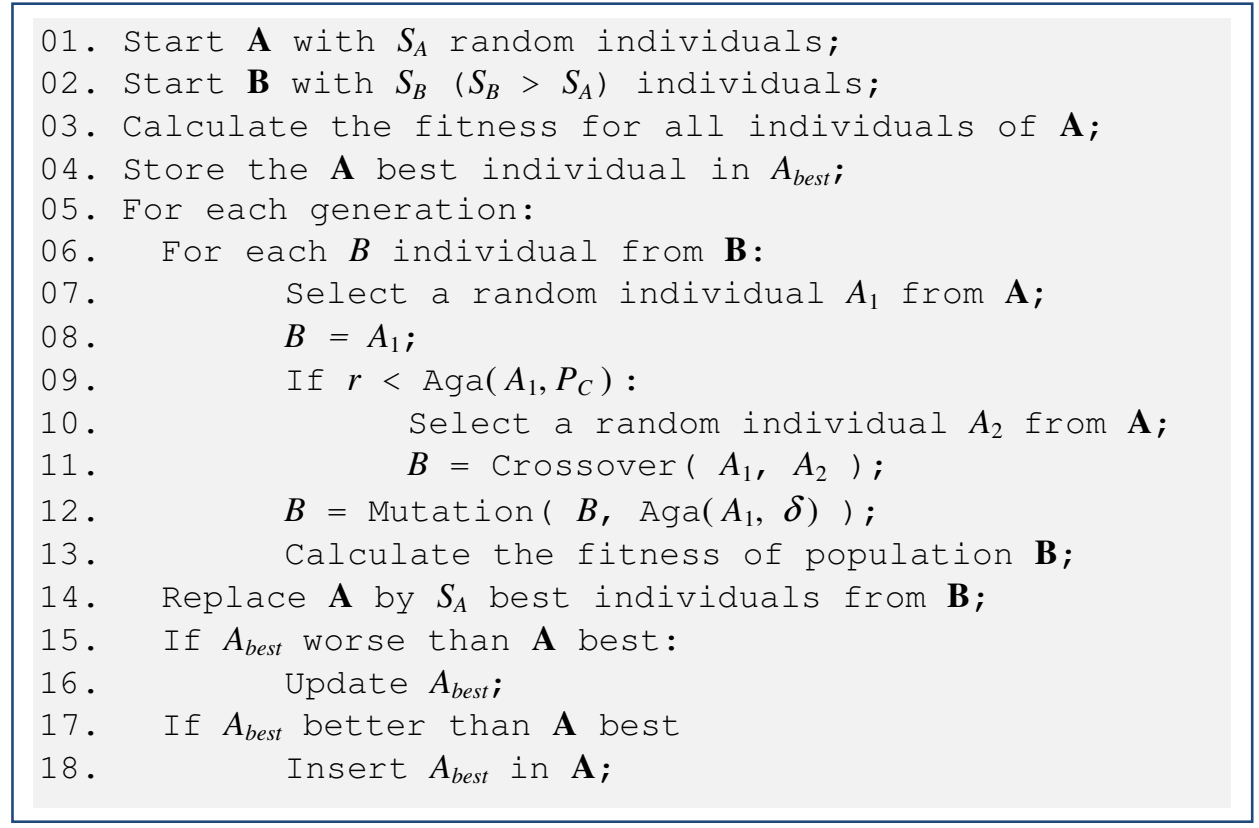

Fig. 2. ES algorithm.

The adapted version of ES used in this article should be better defined as a $\left(S_{A}, S_{B}\right)-$ ES, where $S_{A}$ and $S_{B}$ are respectively the sizes of parent and child populations [19]. The algorithm is shown in Fig. 2. It starts creating two populations, $\mathbf{A}$ and $\mathbf{B}$, to store respectively $S_{A}$ and $S_{B}$ individuals. Inside the generation loop (line 5), each individual of $\mathbf{B}$ is replaced by a new one made by the application of the 
intermediate crossover and Gaussian mutation. $r$ in line 9 represents a uniform random value between 0 and 1. In this same line the function Aga( ) returns the crossover probability based on $A_{1}$ rank, using Adaptive Genetic Algorithm (AGA) procedure defined in [20]. Thus, the intermediate crossover operator is optionally applied in line $11 \mathrm{using} A_{1}$ and $A_{2}\left(A_{2} \neq A_{1}\right)$ as parents. In line 12 the $\mathrm{Aga}()$ function is used again in order to feed Gaussian mutation with its standard deviation [18]. In the line 14 all individuals of population $\mathbf{A}$ are replaced by the $S_{A}$ best individuals copied from $\mathbf{B}$. The iteration is complete after the elitist procedure from 15 to 18 . Note in the line 18 the individual $A_{\text {best }}$ being inserted in $\mathbf{A}$, what set the population size temporally to $S_{A}+1$ until $\mathbf{A}$ be reset in line 14 in the next generation. The fitness calculations in lines 3 and 13 are based on the sum of squared errors between a target curve and the FBG reflectance spectrum. Let $R_{i}$ be the $i$-th reflectance value calculated for wavelength $\lambda_{\mathrm{i}}$ and $T_{i}$ be the respective target value. The fitness value $f$ for each individual can be calculated by

$$
f=\sum_{i=0}^{N-1}\left(R_{i}-T_{i}\right)^{2},
$$

where $N$ represents the number of samples used to build the reflectance curve and $R_{i}$ is calculated using (5). Since the fitness function is an error value, the ES of Fig. 2 minimizes $f$.

The Aga( ) function used in lines 9 and 12 can be defined as

$$
\operatorname{Aga}(A, \alpha)=\left\{\begin{array}{ll}
\alpha \frac{\operatorname{rank}(A)}{h}, & \operatorname{rank}(A)<h \\
\alpha, & \text { otherwise }
\end{array},\right.
$$

where $\operatorname{rank}(A)$ returns the rank of the $A$ individual inside population $\mathbf{A}$ (it returns zero for the best and $\left(S_{A}-1\right)$ for the worst), $h$ is the rank of the individual whose fitness value is closest to the average, $\alpha$ is the control parameter. Aga( ) returns zero for the best ranked individual in the population and $\alpha$ for the worst.

\section{Simulations}

In order to validate the proposed encoding schemes, the ES defined in the previous section was applied using direct encoding, LE, QSE and CSE. All following results were obtained using $S_{A}=10$, $S_{B}=40$, mutation deviation and crossover probability given by AGA using $\alpha$ equals to 0.01 and 1.0 respectively. The maximum number of generations of 10000 was used as stop criteria.

Two projects were used to compare the proposed techniques. The first one was a FBG centered in $1.55 \mu \mathrm{m}$ and the second was a TFBG from $1.5495 \mu \mathrm{m}$ to $1.5505 \mu \mathrm{m}$. The target curves of both are shown in Fig. 3. Note that the target curve for the FBG is not continuous. The interval from $1.5498 \mu \mathrm{m}$ to $1.5502 \mu \mathrm{m}$ has two gaps of $0.05 \mathrm{~nm}$ left and right. They offer accommodation space for reflectance curves once abrupt targets are very difficult to fit.

The fitness in all simulations was calculated using (14) with number of samples $N$ set to 100 . 
Samples are spaced uniformly in terms of wavelength inside the target region. If one sample drops inside a gap in the FBG target, it is not considered in the sum of (14). In all simulations the number of uniform sections used was $50(M=50)$.

For each project and for each encoding, the ES was tested 20 times with different random initial populations.

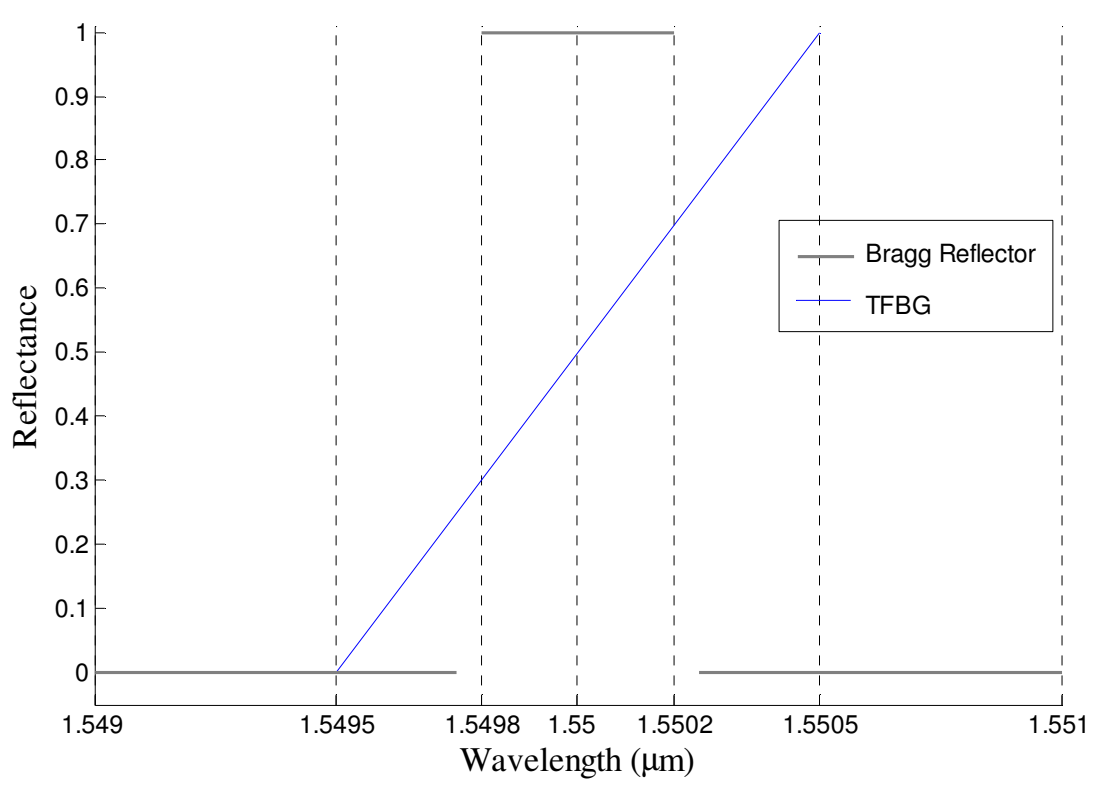

Fig. 1. Target curves for FBG and TFBG projects.

\section{A. FBG project}

For the FBG project, all sections have the same parameters $\Delta z$ and $\lambda_{\mathrm{B}}$ respectively set to $200 \mu \mathrm{m}$ and $1.55 \mu \mathrm{m}$. Indirect encoding schemes used $m=5$ for $\delta n$. Both direct and indirect encodings used $0<\delta n_{k}$ $<5 \times 10^{-4}$.

Fig. 3 compares the $\delta$ profile and reflectance curve for the most ranked Bragg reflectors synthesized using LE, QSE and CSE. The linear interpolation was the worst among all indirect encoding schemes. QSE and CSE schemes gave almost same results. Fig. 4 compares the results obtained for QSE and DE. Direct encoding achieved the best reflectance curve, but the $\delta n$ obtained profile was not as smooth as others obtained using QSE and CSE. It is interesting to observe how all profiles are near each other.

Fig. 5 shows the average evolution of fitness in function of generation number for ES using DE, QSE and CSE. Direct encoding has poor performance since spends more generations to reach convergence. This behavior was expected because here DE dealt with 10 times more dimensions in search space than indirect encodings. The number of generations can be used safely as performance measurement parameter because the fitness function is called exactly 40 times each generation. Furthermore, the computational time spent in interpolations and spline approximations were 
negligible next the processing time spent in fitness calculations.

Table I shows performance parameters: the best final fitness found in all 20 runs, worst fitness found, the mean fitness and the standard deviation. It also shows the convergence number, i.e., the average number of generations necessary to grant fitness value with an error of $0.1 \%$ in comparison to the last fitness value.

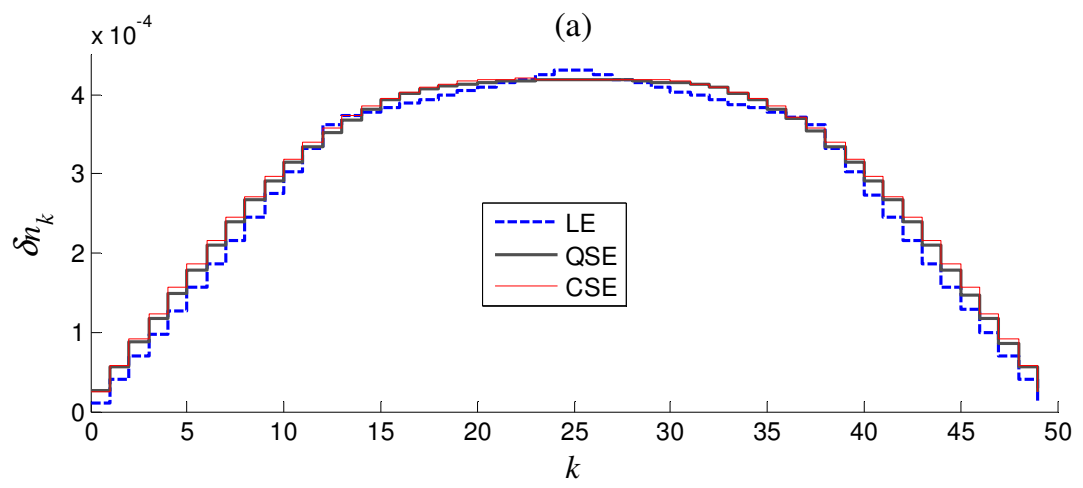

(b)

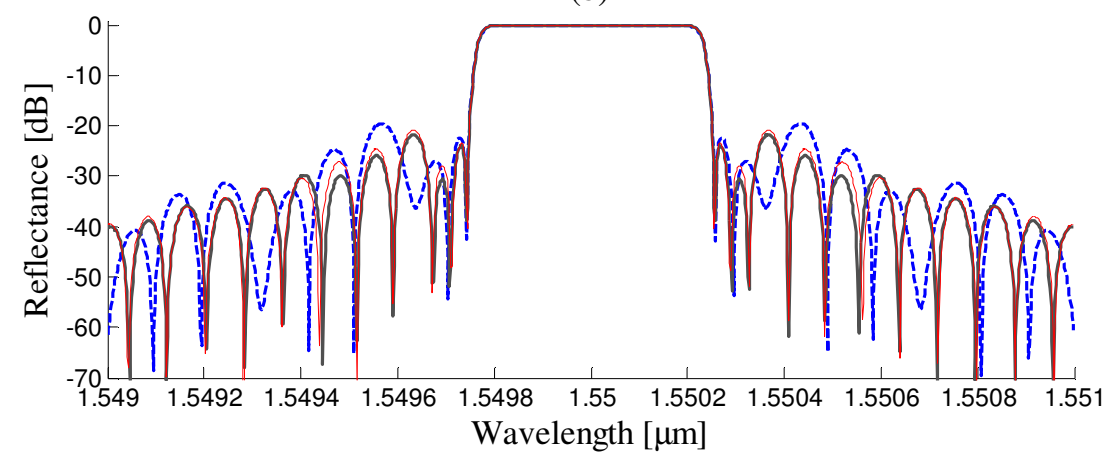

Fig. 2. $\delta n$ profiles (a) and reflectance spectra (b) for FBGs synthesized using LE, QSE and CSE encoding schemes. 


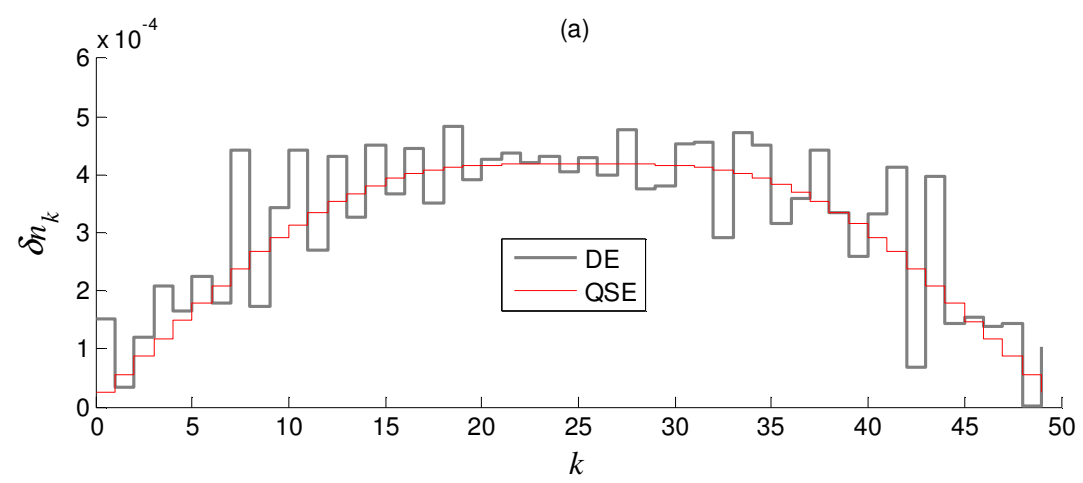

(b)

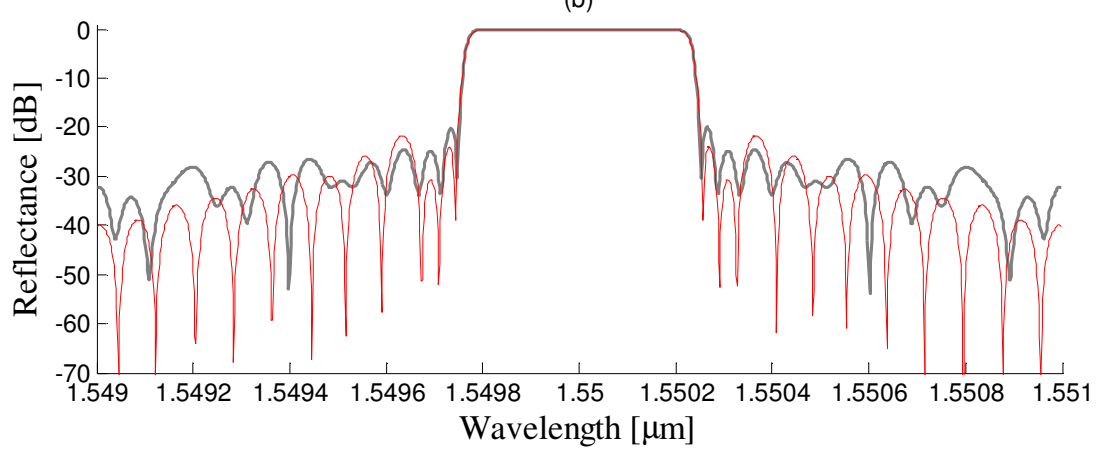

Fig. 3. $\delta$ profiles (a) and reflectance spectrum (b) for FBG synthesized using QSE and DE.

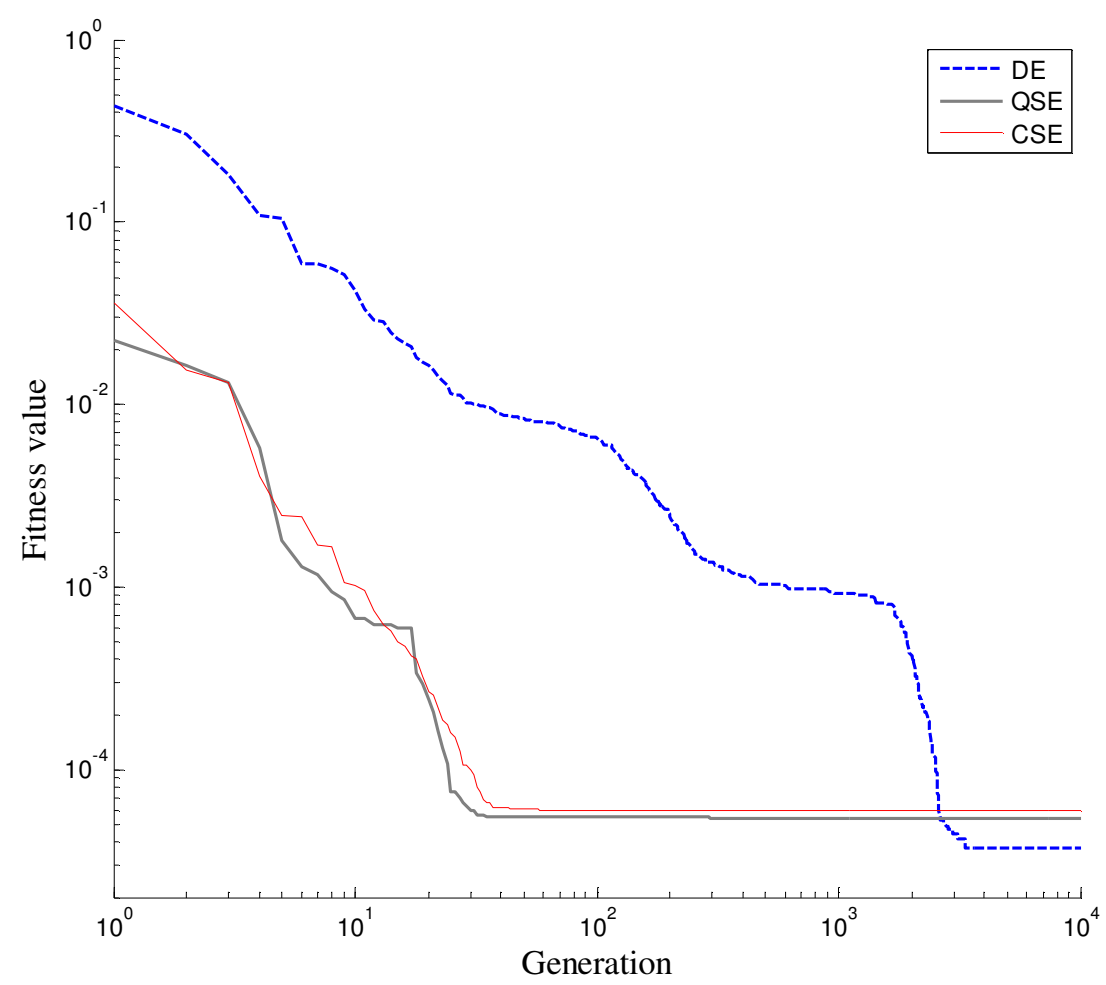

Fig. 6. Average curves of fitness value in function of generation number for QSE, CSE and DE. 
TABLE I. FBG PERFORMANCE PARAMETERS

\begin{tabular}{ccccc}
\hline Parameter & DE & LE & QSE & CSE \\
\hline Best & 0.0286 & 0.05667 & 0.03690 & 0.03870 \\
Mean & 0.0314 & 0.05671 & 0.03692 & 0.03872 \\
Worst & 0.0424 & 0.05674 & 0.03695 & 0.03875 \\
Deviation & $1.76 \times 10^{-5}$ & $1.98 \times 10^{-5}$ & $1.60 \times 10^{-5}$ & $1.63 \times 10^{-5}$ \\
Convergence & 2634 & 208 & 185 & 141 \\
\hline
\end{tabular}

\section{B. TFBG project}

For the TFBG project, all sections used $\Delta z=400 \mu \mathrm{m}$ and linear chirp defined by the variation of $\lambda_{B}$ in function of $k$. As stated in [12], the linear chirp is necessary in order to achieve the desired bandwidth of $1 \mathrm{~nm}$. However, differently from [12], where the linear chirp was fixed, here it was defined by ES itself by encoding $\lambda_{B}{ }_{k}$ in individuals always using LE with $m=2$. The parameter $\delta n_{k}$ was encoded using $m=7$ for indirect encoding schemes. Thus, individuals were represented as a vector of 9 dimensions for indirect encoding schemes and of 52 dimensions for DE. All encoded schemes used $0<\delta n_{k}<5 \times 10^{-4}$ and $1549 \mathrm{~nm}<\lambda_{B k}<1551 \mathrm{~nm}$.

Fig. 7 compares $\delta n$ profiles and reflectance spectra of best ranked FBGs synthesized using LE, QSE and CSE schemes for the TFBG project. Fig. 8 compares only the results obtained by using QSE and DE encodings. As observed in the Bragg reflector project, the linear interpolation brings the worst results among all studied indirect encoding schemes. QSE and CSE are very similar to each other in terms of reflectance but inverted in terms of profile. Indeed, the chirp obtained for DE, LE, QSE and CSE were respectively $1.1536 \mathrm{~nm} / \mathrm{cm}, 1.4206 \mathrm{~nm} / \mathrm{cm}, 1.4254 \mathrm{~nm} / \mathrm{cm}$ and $-1.4188 \mathrm{~nm} / \mathrm{cm}$. The CSE minus signal indicates a decreasing $\lambda_{B}$ toward the last section of the FBG. Since no restrictions were imposed concerning the inclination of $\lambda_{B}$ curves, negative and positive values were obtained evenly for all encodings. Fig. 9 shows the average evolution of the fitness value in function of generation number for ES using direct encoding, LE, QSE and CSE. Table II shows some performance parameters for TFBG project. As observed in the FBG project, the DE resulted again the best among all encoding schemes in terms of fitness function. The DE superiority is not so obvious in terms of reflectance as one can see in Fig. 8. As expected, the optimization process using DE spends more generations to converge. However the difference between DE and indirect encodings is not so deep as observed in the FBG project. 


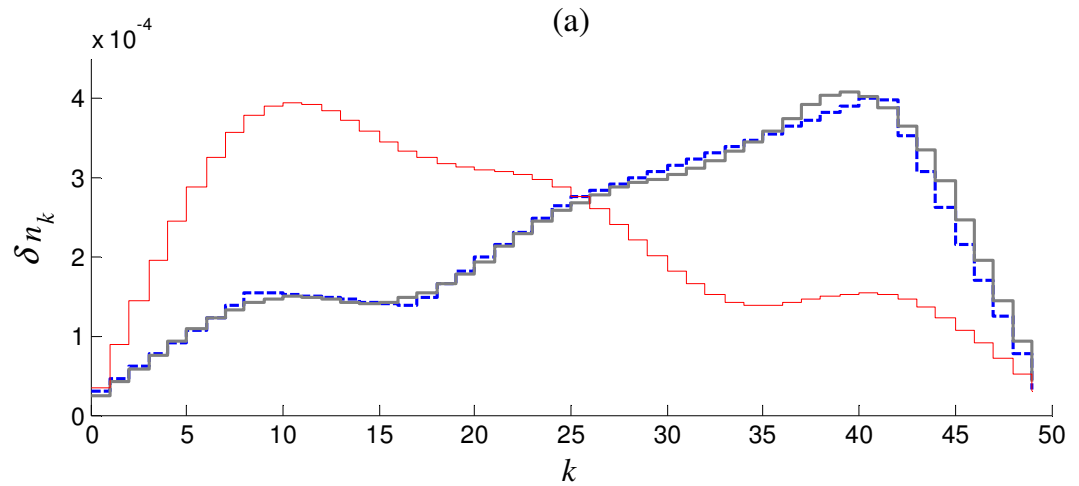

(b)

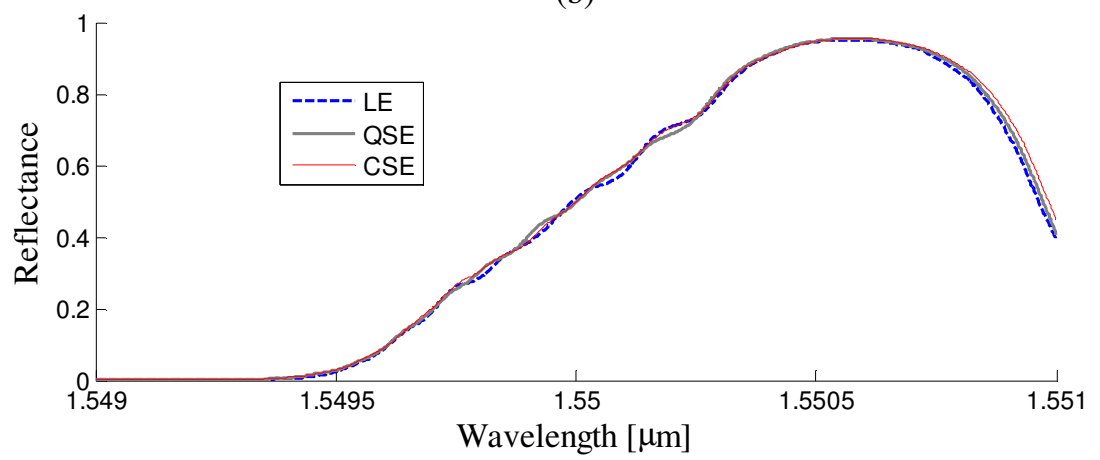

Fig. 7. $\delta n$ profiles (a) and reflectance spectrum (b) for TFBG synthesized using LE, QSE and CSE.

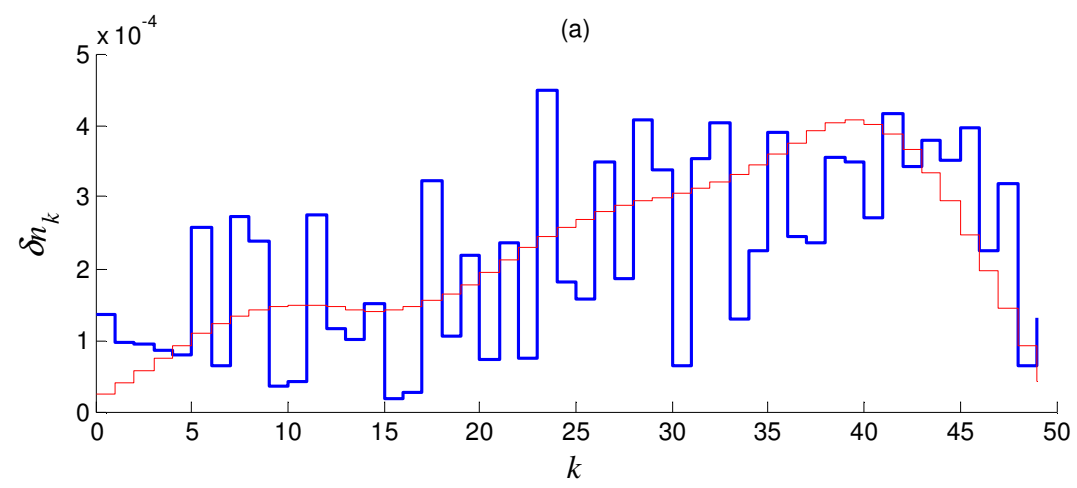

(b)

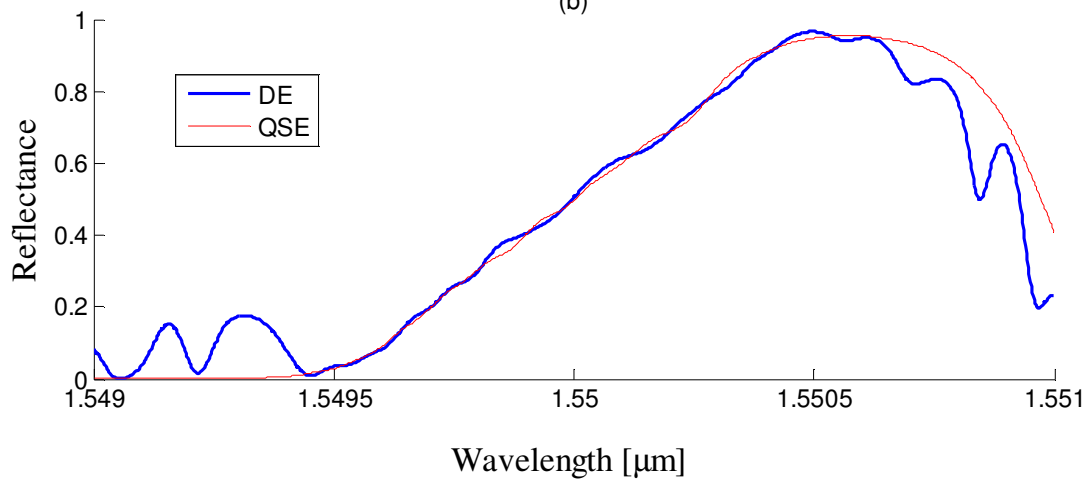

Fig. 8. $\delta$ profiles (a) and reflectance spectrum (b) for TFBG synthesized using QSE and DE. 


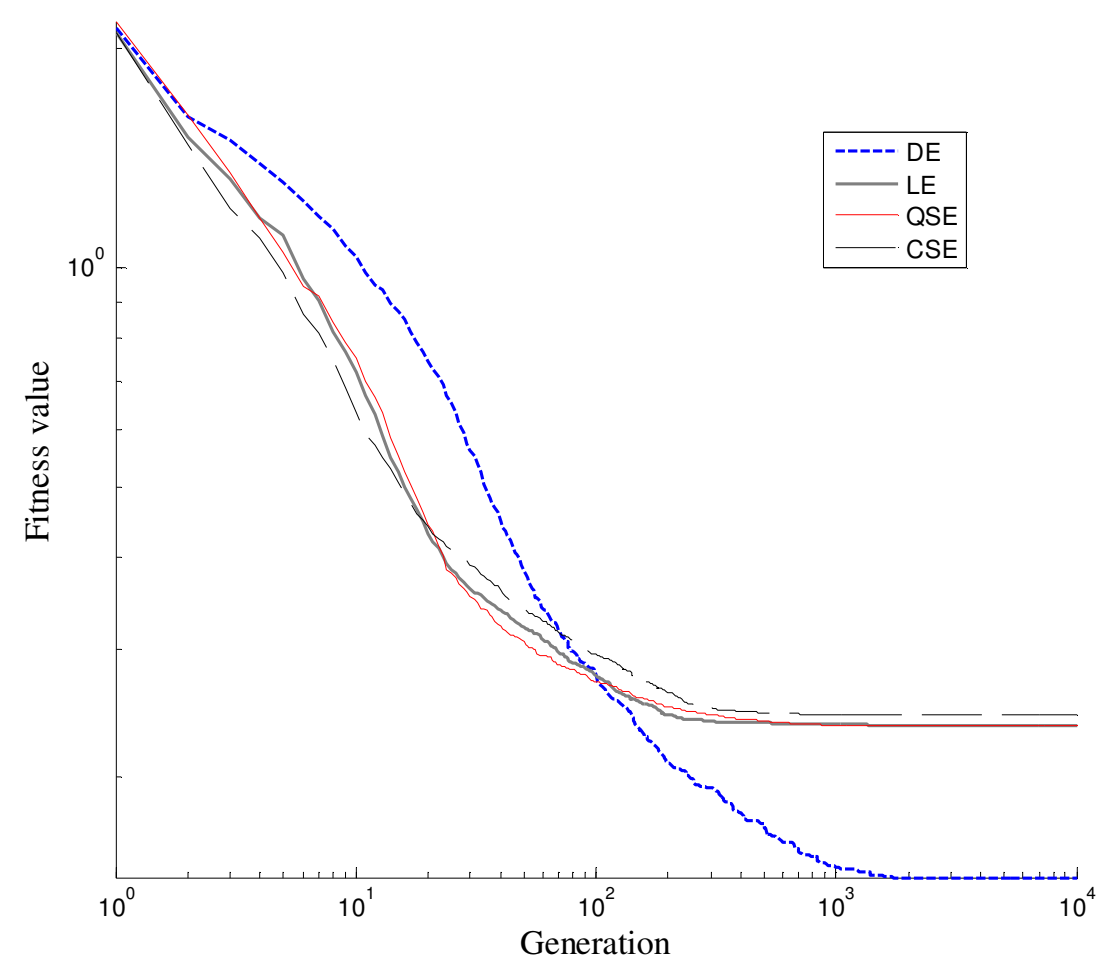

Fig. 9. Average curves of fitness value in function of generation number for DE, LE, QSE and CSE.

TABLE II. TFBG PERFORMANCE PARAMETERS

\begin{tabular}{ccccc}
\hline Parameter & DE & LE & QSE & CSE \\
\hline Best & 0.1218 & 0.1425 & 0.1326 & 0.1339 \\
Mean & 0.1456 & 0.2356 & 0.2354 & 0.2454 \\
Worst & 0.2035 & 0.2585 & 0.2723 & 0.3130 \\
Deviation & 0.0179 & 0.0396 & 0.0597 & 0.0642 \\
Convergence & 872 & 513 & 572 & 492 \\
\hline
\end{tabular}

\section{CONCLUSIONS}

Spline encodings combined to a modified evolutionary strategy have been successfully applied in the FBG synthesis. Two projects have been considered and comparisons involving quadratic and cubic spline encodings, direct encoding, and linear encoding have been provided. It has been shown that spline schemes are able to reduce the number of dimensions and generate attractive softened refractive index profiles.

Quadratic spline encoding (QSE) should always be considered in detriment of cubic encoding (CSE) because it is simpler, has comparable performance and give better results.

However, spline encodings are not free of issues. If the search space is too much simplified, the possible solutions can lose its flexibility. As result, the direct encoding overcame the indirect encoding schemes. Nevertheless the results from spline schemes are more attractive due its simpler 
profiles. An interesting possibility to conciliate performance and flexibility would be to use spline encoding to achieve an initial solution for further application of a direct encoded meta-heuristic or another suitable optimization technique.

The definition of $m$ parameter in indirect encodings was accomplished based on a trial and error procedure. A better way to do that could be through a progressive spline encoding scheme where the number of parameters $m$ would be increased gradually whenever the population diversity drops below a threshold. This technique would require the insertion of new random parameters in individuals in the middle of optimization process, probably in a very similar way as performed in [21].

\section{REFERENCES}

[1] K. A. Winick and J. E. Roman, "Design of corrugated waveguide filters by Fourier transform techniques," IEEE Journal of Quantum Electronics, vol. 26, pp. 1918-1929, 1990.

[2] J. Skaar and O. H. Waagaard, "Design and Characterization of Finite-Length Fiber Gratings," IEEE Journal of Quantum Electronics, vol. 39, pp. 1238-1245, 2003.

[3] L. Ming, H. Junya, and L. Hongpu, "Advanced design of a complex fiber Bragg grating for a multichannel asymmetrical triangular filter," Journal of Optoelectronics and Advanced Materials, pp. 228-234, 2009.

[4] B. Malo, K. O. Hill, F. Bilodeau, D. C. Johnson, and J. Albert, "Point-by-Point Fabrication of Micro-Bragg Gratings in Photosensitive Fiber Using Single Excimer Pulse Refractive-Index Modification Techniques," Electronic Letters, vol. 29, pp. 16681669, 1993.

[5] K. Aksnes and J. Skaar, "Design of short fiber Bragg gratings by optimization," Applied Optics, vol. 43, pp. 2226-2230, 2004.

[6] D. E. Goldberg, Genetic Algorithms in Search, Optimization, and Machine Learning. Addison-Wesley Professional, 1989.

[7] J. Kennedy and R. C. Eberhart, "Particle swarm optimization," Proceedings of IEEE International Conference on Neural Networks, pp. 1942-1948, 1995.

[8] J. Skaar and K. M. Risvik, "A Genetic Algorithm for the Inverse Problem in Synthesis of Fiber Gratings," IEEE Journal of Lightwave Technology, vol. 16, pp. 1928-1932, 1998.

[9] S. Baskar, A. Alphones, P. N. Suganthan, N. Q. Ngo, and R. T. Zheng, "Design of Optimal Length Low-Dispersion FBG Filter Using Covariance Matrix Adapted Evolution," IEEE Photonics Techonology Letters, vol. 17, pp. 2119-2121, 2005.

[10] S. Baskar, R. T. Zheng, A. Alphones, N. Q. Ngo, and P. N. Suganthan, "Particle Swarm Optimization for the Design of Low-Dispersion Fiber Bragg Gratings," IEEE Photonics Techonology Letters, vol. 17, pp. 615-617, 2005.

[11] R. Huang, Y. Zhou, H. Cai, R. Qu, and Z. Fang, "A fiber Bragg grating with triangular spectrum as wavelength readout in sensor systems," Optics Communications, pp. 197201, 2004.

[12] S. Baskar, P. N. Suganthan, N. Q. Ngo, A. Alphones, and R. T. Zheng, "Design of triangular FBG filter for sensor applications using covariance matrix adapted evolution algorithm," Optics Communications, pp. 716-722, 2006.

[13] T. Erdogan, "Fiber grating spectra," Journal of Lightwave Technology, vol. 15, pp. 12771294, 1997. 
[14] D. E. Knuth, Metafont: The Program. Addison Wesley Publishing Company, 1986.

[15] W. H. Press, S. A. Teukolsky, W. T. Vetterling, and B. P. Flannery, Numerical recipes in $C++$, 3rd ed. Cambridge University Express, 2002.

[16] Gerald Farin, Curves and surfaces for computer-aided geometric design. Academic Press, 1996.

[17] T. Bäck and Schwefel, "Evolutionary computation: an overview," Proceedings of IEEE International Conference on Evolutionary Computation, pp. 20 - 29, 1996.

[18] Z. Michalewicz, Genetic Algorithms + Data Structures = Evolution Programs. Springer-Verlag, 1992.

[19] T. B. and H.-P. Schwefel, "Evolutionary computation: comments on the history and current state," IEEE Transactions on Evolutionary Computation, vol. 1, pp. 3-17, 1997.

[20] Z. Liu, J. Zhou, and S. Lai, "New adaptive genetic algorithm based on ranking," International Conference on Machine Learning and Cybernetics, pp. 1841-1844, 2003.

[21] Il Yong Kim and Olivier de Weck. "Variable Chromosome Length Genetic Algorithm for Progressive Refinement in Topology Optimization," Structural and Multidisciplinary Optimization, Vol. 29, pp. 445-456, 2005. 